\title{
$\mathrm{HNCO} \stackrel{h v}{\longrightarrow} \mathrm{HN}+\mathrm{CO}$ 势能面研究 ${ }^{*}$
}

\section{赵丽李宗和 ${ }^{* *}$}

(北京师范大学化学系, 北京 100875)

\begin{abstract}
摘要用从头算方法, 在 $\mathrm{UHF} / 6-311 \mathrm{G}^{* *}$ 和 CIS/6-311G**水平上, 分别考察了异氭酸 光解反应 $\mathrm{HNCO} \stackrel{h v}{\longrightarrow} \mathrm{HN}+\mathrm{CO}$ 在基态 $\left(\mathrm{S}_{0}\right)$ 、第一激发三态 $\left(\mathrm{T}_{1}\right)$ 和第一激发单态 $\left(\mathrm{S}_{1}\right)$ 势 能面上的解离曲线. 并找到了 $\mathrm{S}_{1} / \mathrm{T}_{1}, \mathrm{~T}_{1} / \mathrm{S}_{0}, \mathrm{~S}_{1} / \mathrm{S}_{0}$ 势能面的交叉点, 给出了极小能量交叉 点的特征，在此基础上根据梯度最速下降原理确定了态-态跃迁后交叉点构型在低能态 势能面的变化及归宿。计算结果表明光解反应 $\mathrm{HNCO} \stackrel{h v}{\longrightarrow} \mathrm{HN}+\mathrm{CO}$ 存在三条竞争反 应途径, 从动力学观点看, 途径 $\mathrm{A}$ 对反应最为有利.
\end{abstract}

\section{关键词 异氰酸 光解 势能面交叉}

异氞酸在燃烧和大气化学中起着重要的作用, 它可以从燃烧的废气中快速除去有毒的 $\mathrm{NO}_{x}$ 化合物 $\left(\mathrm{RAPRENO}_{x}\right)^{[1]}$, 高能燃料的燃烧也涉及到异氭酸 ${ }^{[2]}$, 此外异氭酸还是实验室中用 于研究基元反应动力学的 $\mathrm{NCO}$ 和 $\mathrm{NH}$ 基团的便利的光解源 ${ }^{[2]}$, 所以, 异氧酸的光解反应一直 是光化学领域比较感兴趣的课题之一.

虽然异氧酸只是一个四原子的分子, 但揭示它的光解反应机理却是一个十分复杂的过程, 因为它至少涉及到 3 个势能面(基态 $\left(\mathrm{S}_{0}\right)$, 第一激发三态 $\left(\mathrm{T}_{1}\right)$, 第一激发单态 $\left.\left(\mathrm{S}_{1}\right)\right)$ 和 3 个相互竞 争的反应通道 ${ }^{[3]}$ :

$$
\begin{array}{ll}
\mathrm{HNCO} \rightarrow \mathrm{NH}\left(\mathrm{X}^{3} \Sigma^{-}\right)+\mathrm{CO}\left(\mathrm{X}^{1} \Sigma^{+}\right) & \mathrm{R}(1) \\
\mathrm{HNCO} \rightarrow \mathrm{H}\left({ }^{2} \mathrm{~S}\right)+\mathrm{NCO}\left(\mathrm{X}^{2} \Pi\right) & \mathrm{R}(2) \\
\mathrm{HNCO} \rightarrow \mathrm{NH}\left(\mathrm{a}^{1} \Delta\right)+\mathrm{CO}\left(\mathrm{X}^{1} \Sigma^{+}\right) & \mathrm{R}(3)
\end{array}
$$

近年来许多研究小组用不同的实验方法对异氧酸的紫外光解进行了广泛的研究, 主要将 焦点集中在异氭酸的选键光解和 $\mathrm{S}_{1}$ 态光解反应产物 $\mathrm{NCO}$ 和 $\mathrm{NH}$ 的分支比 $\phi_{\mathrm{NCO}} / \phi_{\mathrm{NH}}$ 的测定 ${ }^{[4,5]}$, 在理论上, Morokuma 等在 $\mathrm{sa}-\operatorname{CASSCF}(12 \mathrm{e} / 10 \mathrm{o}) / \mathrm{d} 95++$ 水平上确定了异氧酸在 $\mathrm{S}_{1}$ 势能面上的驻 点 ${ }^{[6]}$, Fang 等计算了异氭酸 $\mathrm{C}-\mathrm{N}$ 键解离的一维势能曲线 ${ }^{[7]}$, Brown 等计算了一个二维的激发态 势能面(两个自由度分别为 $R_{\mathrm{NH}}$ 和 $R_{\mathrm{CN}}$ ), 并做了传统的轨线计算, 再现了反应分支比随波长的 变化及 $v_{\mathrm{NH}}=3$ 时的选键性 ${ }^{[8]}$. Klossica 等给出了异氰酸 $\mathrm{S}_{1}\left({ }^{1} \mathrm{~A}^{\prime \prime}\right)$ 态三维势能面的计算结果, 讨论 了生成 $\mathrm{HN}\left(\mathrm{a}^{1} \Delta\right)+\mathrm{CO}\left(\mathrm{X}^{1} \Sigma^{+}\right)$和 $\mathrm{H}\left({ }^{2} \mathrm{~S}\right)+\mathrm{NCO}\left({ }^{1} \mathrm{~A}^{\prime \prime}\right)$ 这两个反应通道 ${ }^{[9]}$, 但没有涉及反应通道 $\mathrm{R}(1)$. 而对 $\mathrm{HNCO} \rightarrow \mathrm{HN}+\mathrm{CO}$ 这个反应, Zyrianov 等推测它最可能的解离途径是 $\mathrm{S}_{1} \rightarrow \mathrm{S}_{0} \rightarrow \mathrm{T}_{1} \rightarrow{ }^{3} \mathrm{NH}+$ $\mathrm{CO}^{[10]}$, 但相关的理论研究一直未见报道.

本文的目的是要从理论上研究此反应是否与三态相关, 我们首先分别用 CIS/6-311G**, 
$\mathrm{UHF} / 6-311 \mathrm{G}^{* *}$ 的方法研究了激发单态 $\left(\mathrm{S}_{1}\right)$ 和激发三态 $\left(\mathrm{T}_{1}\right)$ 的势能面, 然后用 Lagrange-Newton 方法找到了 $\mathrm{S}_{1} / \mathrm{T}_{1}, \mathrm{~T}_{1} / \mathrm{S}_{0}$ 极小能量交叉点, 并在反式解离的 $\mathrm{S}_{1}$ 最小能量途径线上找到 $\mathrm{S}_{1} / \mathrm{S}_{0}$ 交叉 点, 根据梯度最速下降原理确定了态-态跃迁后交叉点构型在低能态势能面的变化及归宿, 计 算结果表明光解反应最有利的通道是由激发单态 $\left(S_{1}\right)$ 向三态 $\left(T_{1}\right)$ 跃迁, 然后沿三态进行的, 最 后生成三态的产物, 与 Zyrianov 推测的产物相同 ${ }^{[10]}$. 整个工作在 P II -300 微机上完成.

\section{$1 \mathrm{HNCO} \rightarrow \mathrm{HN}+\mathrm{CO}$ 势能剖面}

用 Gaussian94 程序在 UHF/6-311G**水平上优化了异氭酸基态 $\left(\mathrm{S}_{0}\right)$ 和第一激发态三态 $\left(\mathrm{T}_{1}\right)$ 的平衡几何构型(见表 1), 并考察了这两个态 $\mathrm{C}-\mathrm{N}$ 键的解离情况, 做出了 $\mathrm{S}_{0}$ 和 $\mathrm{T}_{1}$ 态的解离曲 线(分别见图 1 和 2 ), 发现 $\mathrm{S}_{0}$ 态是非解离态, $\mathrm{T}_{1}$ 态是预解离态, 在此基础上确定了 $\mathrm{T}_{1}$ 态反应途径上 的驻点(反应物, 产物, 过渡态)(见表 1 及 2), 并对过渡态进行了确认(惟一的虚频 $\omega=-695.9147 \mathrm{i}$, 且振动模式正方向指向产物, 反方向指向反应物).

表 $1 \mathrm{~S}_{0}, \mathrm{~T}_{1}$ 态反应物、过渡态的几何构型优化值和能量(UHF/6-311G**)

\begin{tabular}{lccc}
\hline & $\mathrm{HNCO}\left(\mathrm{S}_{0}\right)$ & $\mathrm{HNCO}\left(\mathrm{T}_{1}\right)$ & $\mathrm{TS}\left(\mathrm{T}_{1}\right)$ \\
\hline$R_{\mathrm{NH}} / \mathrm{nm}$ & 0.0994 & 0.1011 & 0.1015 \\
$R_{\mathrm{CN}} / \mathrm{nm}$ & 0.1197 & 0.1423 & 0.1693 \\
$R_{\mathrm{CO}} / \mathrm{nm}$ & 0.1139 & 0.1155 & 0.1129 \\
$\angle \mathrm{HNC} /\left(^{\circ}\right)$ & 127.78 & 108.03 & 103.2559 \\
$\angle \mathrm{NCO} /\left(^{\circ}\right)$ & 174.65 & 124.47 & 121.5471 \\
二面角 $/\left(^{\circ}\right)$ & 180.0 & 138.73 & 180 \\
能量/hartree & -167.8104072 & -167.7095279 & -167.7019235 \\
\hline
\end{tabular}

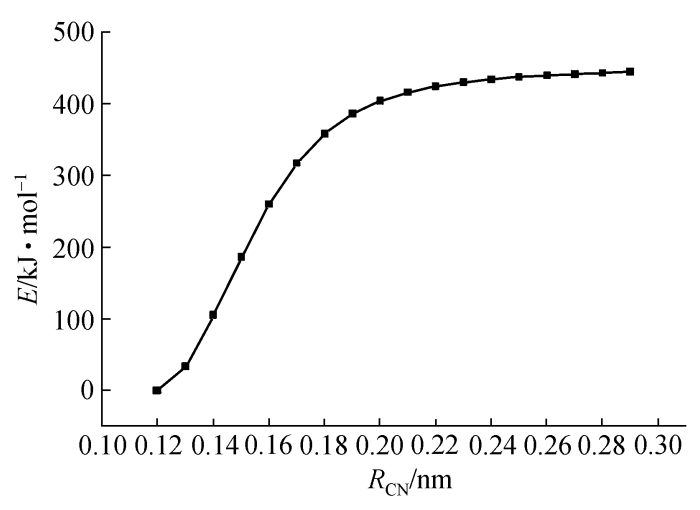

图 $1 \quad \operatorname{HNCO}\left({ }^{1} \mathrm{~A}^{\prime}\right)=\mathrm{HN}\left(\mathrm{b}^{1} \Sigma^{+}\right)+\mathrm{CO}\left(\mathrm{X}^{1} \Sigma^{+}\right)$

$\mathrm{S}_{0}$ 解离势能曲线

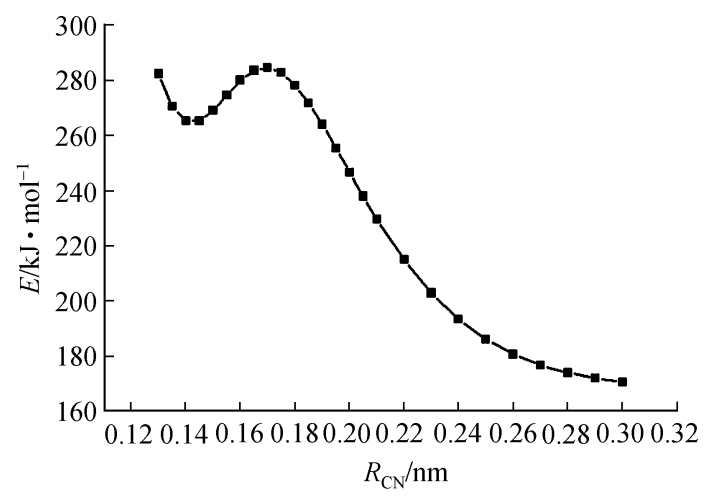

图 $\left.2 \mathrm{HNCO}^{3} \mathrm{~A}^{\prime \prime}\right)=\mathrm{HN}\left(\mathrm{X}^{3} \Sigma^{+}\right)+\mathrm{CO}\left(\mathrm{X}^{1} \Sigma^{+}\right)$ $\mathrm{T}_{1}$ 态解离势能曲线

用 Gaussian94 程序在 CIS/6-311G**水平上考察了第一激发单态 $\left(\mathrm{S}_{1}\right)$ 的反应, 发现异㲵酸的 $\mathrm{S}_{1}$ 态有顺反两种平衡几何构型(见表 3 ). 分别作出了 $\mathrm{S}_{1}$ 态顺式反应和反式反应的解离曲线(见 图 3), 都是预解离线, 驻点构型见表 3 及表 2 , 并确定了这两条反应途径上的过渡态和顺反异 构化的过渡态及其转化途径(如图 4), 过渡态构型见表 3 . 
表 $2 \mathrm{R}(1)$ 和 $\mathrm{R}(3)$ 反应产物的构型和能量

\begin{tabular}{lcc}
\hline & 键长/nm & 能量/hartree \\
\hline $\mathrm{NH}\left(\mathrm{a}^{1} \Delta\right)\left(\mathrm{CIS} / 6-311 \mathrm{G}^{* *}\right)$ & 0.1020 & -54.901081 \\
$\mathrm{NH}\left(\mathrm{X}^{3} \Sigma^{-}\right)\left(\mathrm{UHF} / 6-311 \mathrm{G}^{* *}\right)$ & 0.1023 & -54.9760629 \\
$\mathrm{CO}\left(\mathrm{X}^{1} \Sigma^{+}\right)\left(\mathrm{UHF} / 6-311 \mathrm{G}^{* *}\right)$ & 0.1105 & -112.7694753 \\
\hline
\end{tabular}

表 $3 \mathrm{~S}_{1}$ 态反应物、过渡态的构型、能量 $(\mathrm{CIS} / 6-311 \mathrm{G} * *)$

\begin{tabular}{lccccc}
\hline & $\mathrm{HNCO}\left(\mathrm{S}_{1}\right.$-反式 $)$ & $\mathrm{HNCO}\left(\mathrm{S}_{1}\right.$-顺式 $)$ & $\mathrm{TS}$ (反式 $)$ & $\mathrm{TS}($ 顺式 $)$ & $\mathrm{TS}($ 顺反异构 $)$ \\
\hline$R_{\mathrm{NH}} / \mathrm{nm}$ & 0.1006 & 0.1020 & 0.1011 & 0.1018 & 0.0990 \\
$R_{\mathrm{CN}} / \mathrm{nm}$ & 0.1362 & 0.1324 & 0.1590 & 0.1583 & 0.1282 \\
$R_{\mathrm{CO}} / \mathrm{nm}$ & 0.1161 & 0.1172 & 0.1131 & 0.1132 & 0.1187 \\
$\angle \mathrm{HNC} /\left(^{\circ}\right)$ & 109.79 & 107.70 & 105.15 & 98.19 & 176.58 \\
$\angle \mathrm{NCO} /\left(^{\circ}\right)$ & 128.32 & 132.51 & 123.04 & 122.69 & 132.63 \\
二面角 $/\left(^{\circ}\right)$ & 180.0 & 0.0 & 180.0 & 0.0 & 0.0 \\
能量 $/$ hartree & -167.6255523 & -167.6141518 & -167.620066 & -167.6017162 & -167.5726675 \\
\hline
\end{tabular}

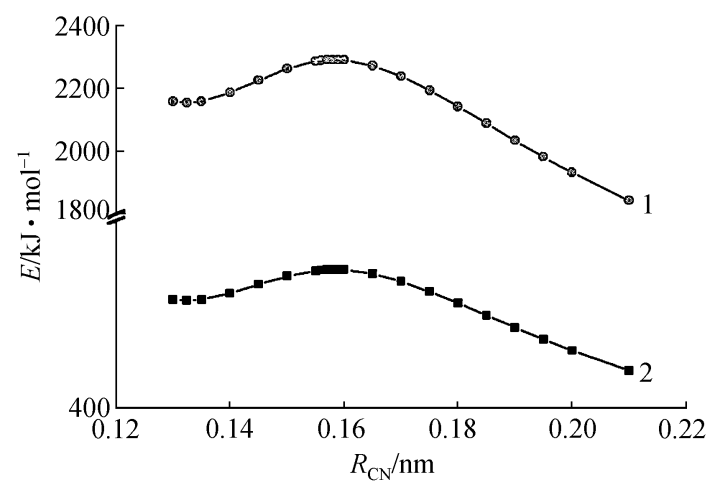

图 $3 \quad \mathrm{HNCO}\left({ }^{1} \mathrm{~A}^{\prime \prime}\right) \rightarrow \mathrm{HN}\left(\mathrm{a}^{1} \Delta\right)+\mathrm{CO}\left(\mathrm{X}^{1} \Sigma\right)$ $\mathrm{S}_{1}$ 顺式(1)和反式(2)解离曲线

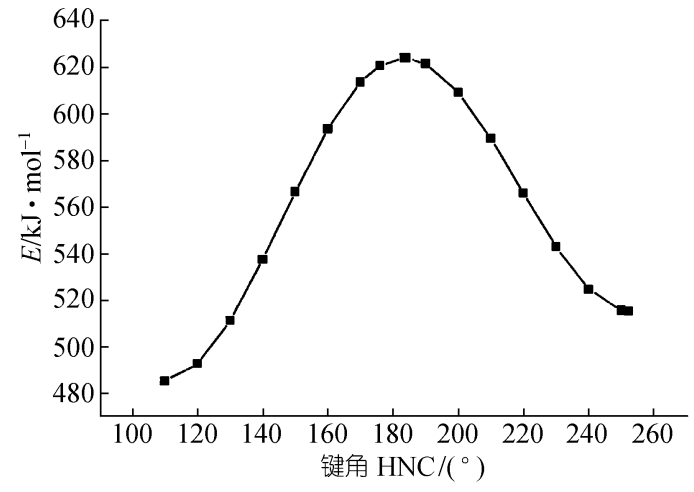

图 $4 \mathrm{~S}_{1}$ 态顺反平衡构型异构化势能曲线

\section{$2 \mathrm{HNCO} \rightarrow \mathrm{HN}+\mathrm{CO}$ 势能面交叉}

在光化学反应中研究势能面交叉不可避免，但 2 个势能面交叉点有无穷多个，而 2 个不同 电子态势能面的极小能量交叉点仅有一个，它在光化学反应中具有极其重要的意义. 根据 Morokuma 使用的 Lagrange-Newton 方法 ${ }^{[11]}$ 寻找势能面极小交叉点, 用自编程序, 从某一个态 的构型出发, 我们找到了势能面 $\mathrm{S}_{1}$ 与 $\mathrm{T}_{1}, \mathrm{~T}_{1}$ 与 $\mathrm{S}_{0}$ 的极小能量交叉点 $\mathrm{S}_{1} / \mathrm{T}_{1}, \mathrm{~T}_{1} / \mathrm{S}_{0}$.

每一步分子构型的变化为

$$
\left(\begin{array}{c}
\delta_{R} \\
\lambda^{K+1}
\end{array}\right)=\left(\begin{array}{cc}
\omega_{K} & -g_{1}^{(K)}+g_{2}^{(K)} \\
-g_{1}^{(K)}+g_{2}^{(K)} & 0
\end{array}\right)^{-1}\left(\begin{array}{c}
-g_{1}^{(K)} \\
E_{1}\left(R_{K}\right)-E_{2}\left(R_{K}\right)
\end{array}\right),
$$

其中, $\lambda$ 为 Lagrange 参量, $K$ 为找寻步数, $\delta_{R}$ 为构型变化, $E_{1}, E_{2}$ 为构型为 $R_{K}$ 时 2 个态所对应的 能量, $g_{1}, g_{2}$ 为 2 个态所对应的梯度,

$$
\omega_{K}=\nabla_{R}^{2} E_{1}\left(R^{K}\right)-\lambda^{K}\left[\left(\nabla_{R}^{2} E_{1}\left(R^{K}\right)-\nabla_{R}^{2} E_{2}\left(R^{K}\right)\right] .\right.
$$

交叉点的构型分别见表 4 和 5.

对 $\mathrm{S}_{1} / \mathrm{T}_{1}, \mathrm{~T}_{1} / \mathrm{S}_{0}$ 交叉点考察的结果表明，这些交叉点除了能量相等外还有如下 3 个特征:

（1）交叉点构型在 2 个态的平衡几何构型之间(见表 4 和 6). 
（2）交叉点处两个态的梯度成比例，且等于 $-\lambda /(1-\lambda)$ (见表 5 和 7 ).

表 $4 \mathrm{~S}_{1} / \mathrm{T}_{1}$ 的构型与 $\mathrm{S}_{1}, \mathrm{~T}_{1}$ 态平衡几何构型的比较

\begin{tabular}{lccr}
\hline & $\mathrm{S}_{1}$ 平衡构型 $\left(\mathrm{CIS} / 6-311 \mathrm{G}^{* *}\right)$ & $\mathrm{S}_{1} / \mathrm{T}_{1}$ 构型 & $\mathrm{T}_{1}$ 平衡构型 $\left(\mathrm{CIS} / 6-311 \mathrm{G}^{* *}\right)$ \\
\hline$R_{\mathrm{NH}} / \mathrm{nm}$ & 0.1006 & 0.1009 & 0.1011 \\
$R_{\mathrm{CN}} / \mathrm{nm}$ & 0.1361 & 0.1374 & 0.1438 \\
$R_{\mathrm{CO}} / \mathrm{nm}$ & 0.1162 & 0.1159 & 0.1148 \\
$\angle \mathrm{HNC} /\left(^{\circ}\right)$ & 109.8253 & 105.2576 & 108.9089 \\
$\angle \mathrm{NCO} /\left(^{\circ}\right)$ & 128.3773 & 128.6146 & 124.0515 \\
二面角 $/\left(^{\circ}\right)$ & 180.0068 & 179.9982 & -84.6474 \\
$E / \mathrm{hartree}$ & & -167.6255546 & \\
\hline
\end{tabular}

表 5 极小能量交叉点 $S_{1} / T_{1}$ 对应于 $S_{1}$ 和 $T_{1}$ 态的梯度及其比值

\begin{tabular}{lcccc}
\hline & $g\left(\mathrm{~S}_{1}\right)$ & $g\left(\mathrm{~T}_{1}\right)$ & $g\left(\mathrm{~S}_{1}\right) / g\left(\mathrm{~T}_{1}\right)$ & $-\lambda /(1-\lambda)^{\mathrm{a})}$ \\
\hline$R_{\mathrm{NH}} / \mathrm{nm}$ & -0.00102 & -0.00676 & 0.1509 & 0.1511 \\
$R_{\mathrm{CN}} / \mathrm{nm}$ & -0.00136 & -0.00914 & 0.1488 & \\
$R_{\mathrm{CO}} / \mathrm{nm}$ & 0.00057 & 0.00368 & 0.1549 & \\
$\angle \mathrm{HNC} /\left(^{\circ}\right)$ & 0.01508 & 0.09997 & 0.1508 & \\
$\angle \mathrm{NCO} /\left(^{\circ}\right)$ & -0.00039 & -0.00271 & 0.1439 & - \\
二面角 $/\left(^{\circ}\right)$ & 0.0 & 0.0 & & \\
\hline
\end{tabular}

a) 最后一步的 $\lambda$ 值为 -0.178

表 $6 \mathrm{~T}_{1} / \mathrm{S}_{0}$ 构型与 $\mathrm{S}_{0}, \mathrm{~T}_{1}$ 态平衡几何构型的比较

\begin{tabular}{lccc}
\hline & $\mathrm{S}_{0}$ 平衡构型 $\left(\mathrm{UHF} / 6-311 \mathrm{G}^{* *}\right)$ & $\mathrm{T}_{1} / \mathrm{S}_{0}$ 构型 & $\mathrm{T}_{1}$ 平衡构型 $\left(\mathrm{UHF} / 6-311 \mathrm{G}^{* *}\right)$ \\
\hline$R_{\mathrm{NH}} / \mathrm{nm}$ & 0.0994 & 0.1009 & 0.1011 \\
$R_{\mathrm{CN}} / \mathrm{nm}$ & 0.1197 & 0.1410 & 0.1423 \\
$R_{\mathrm{CO}} / \mathrm{nm}$ & 0.1139 & 0.1156 & 0.1156 \\
$\angle \mathrm{HNC} /\left(^{\circ}\right)$ & 127.7787 & 108.4735 & 108.0268 \\
$\angle \mathrm{NCO} /\left(^{\circ}\right)$ & 174.6513 & 127.4254 & 124.4685 \\
二面角 $/\left(^{\circ}\right)$ & 180.0 & 165.6424 & 138.7279 \\
$E /$ hartree & & -167.7092224 & \\
\hline
\end{tabular}

表 7 极小能量交叉点 $\mathrm{T}_{1} / \mathrm{S}_{0}$ 对应于 $\mathrm{T}_{1}$ 和 $\mathrm{S}_{0}$ 态的梯度及其比值

\begin{tabular}{lcccc}
\hline & $g\left(\mathrm{~T}_{1}\right)$ & $g\left(\mathrm{~S}_{0}\right)$ & $g\left(\mathrm{~T}_{1}\right) / g\left(\mathrm{~S}_{0}\right)$ & $-\lambda /(1-\lambda)^{\mathrm{a})}$ \\
\hline$R_{\mathrm{NH}} / \mathrm{nm}$ & - & -0.00005 & - & -0.4603 \\
$R_{\mathrm{CN}} / \mathrm{nm}$ & 0.00438 & -0.09560 & -0.04582 & \\
$R_{\mathrm{CO}} / \mathrm{nm}$ & 0.00262 & -0.05711 & -0.04588 & \\
$\angle \mathrm{HNC} /\left(^{\circ}\right)$ & -0.00081 & 0.01771 & -0.04575 & \\
$\angle \mathrm{NCO} /\left(^{\circ}\right)$ & -0.00710 & 0.15458 & -0.04593 & \\
二面角 $/\left(^{\circ}\right)$ & -0.00019 & 0.00417 & -0.04556 & \\
\hline
\end{tabular}

a) 最后一步的 $\lambda$ 值为 0.044

这两个特征和 Morokuma 给出的范例相同 ${ }^{[11]}$, 据此我们可以认为 $\mathrm{S}_{1} / \mathrm{T}_{1}, \mathrm{~T}_{1} / \mathrm{S}_{0}$ 是 2 个不同 态的邻近势能面极小能量交叉点.

（3）通过固定 $\mathrm{N}-\mathrm{C}$ 键键长在 $\mathrm{S}_{1}$ 态下优化 $\mathrm{HNCO}$ 构型发现和交叉点构型一样, 说明 $\mathrm{S}_{1} / \mathrm{T}_{1}$ 构型在反式 $S_{1}$ 态 $N-C$ 键断裂的最小途径上. 它接近 $S_{1}$ 态平衡几何构型, 位于过渡态之前, 直 接影响反应通道. 同理, $\mathrm{T}_{1} / \mathrm{S}_{0}$ 构型在 $\mathrm{T}_{1}$ 态 $\mathrm{N}-\mathrm{C}$ 键缩短方向上的最小能量途径上, 它在 $\mathrm{T}_{1}$ 态 平衡构型之前, 对 $\mathrm{N}-\mathrm{C}$ 键断裂反应影响不大. 这说明极小能量交叉点处在高能态势能面的最 小能量途径上. 这就使得寻找极小能量交叉点更具有特殊意义, 它是化学反应从高能态的势 
能面进人低能态的关键点.

另外, 我们在 $S_{1}$ 的最小能量途径线上寻找到了 $S_{1}$ 态和 $S_{0}$ 态的在同一构型下对应的能量差 值 $\Delta E$ 最小的点(此点对应于 $S_{1}$ 态和 $S_{0}$ 态的能量分别为-167.6416842 和-167.6416857 hartree), $\Delta E=1.5 \times 10^{-6}$ hartree, 它位于过渡态之后, 接近产物, 虽然这个交点不具有这两个势能面的 极小能量交点的特征(1)和(2), 但它位于 $S_{1}$ (反式)的极小能量途径上, 这对于此反应的动力学 研究是有意义的, 反应有可能经由此点实现由 $\mathrm{S}_{1}$ 态向 $\mathrm{S}_{0}$ 态的跃迁，直接影响产物生成. 交叉 点的构型见表 8 .

表 $8 \quad \mathrm{~S}_{1}$ 与 $\mathrm{S}_{0}$ 交点的构型

\begin{tabular}{cccccc}
\hline$R_{\mathrm{NH}} / \mathrm{nm}$ & $R_{\mathrm{NC}} / \mathrm{nm}$ & $R_{\mathrm{CO}} / \mathrm{nm}$ & $\angle \mathrm{HNC} /\left(^{\circ}\right)$ & $\angle \mathrm{NCO} /\left(^{\circ}\right)$ & 二面角 $/\left(^{\circ}\right)$ \\
\hline 0.10169 & 0.19964 & 0.11099 & 100.2642 & 116.4189 & 180.0 \\
\hline
\end{tabular}

\section{3 交叉点结构在低势能面的归宿}

\section{1 确定交叉点的结构在低势能面的变化及归宿}

势能面相交最小能量构型分子在低电子态势能面上经原子核的弛豫振动构型不断演化, 构型变化应服从最速梯度下降原理：

$$
\frac{\mathrm{d} x_{i}}{\left(-\frac{\partial E}{\partial x_{i}}\right)}=\tau, \quad i=1,2, \cdots, 3 N-6,
$$

其中, $\mathrm{d} x_{i}$ 为第 $i$ 个坐标的变化, $\partial E / \partial x_{i}=g_{i}$ 为第 $i$ 个坐标的能量梯度.

当某一构型为 $R^{m}$, 且 $g_{i}^{m}=0(i=1,2, \cdots, 3 n-6)$ 时, 对这一构型作振动分析, 若: 频率 (1) $\omega_{1}, \omega_{2} \cdots \omega_{3 n-6}>0$, 则此点为势能面极小点; (2) 出现 $n$ 个虚频, 则此点为势能面上的 $n$ 阶鞍 点, 这点构型沿每一个虚振动模式还会各自走向势能面极小点.

\section{2 计算结果}

使用上述原理，我们用自编程序找到各交叉点在低电子态势能面上的变化及归宿， $\mathrm{S}_{1} / \mathrm{T}_{1}$ 交点构型在 $\mathrm{T}_{1}$ 势能面上最终落到 $\mathrm{T}_{1}$ 态平衡构型位置上(见图 5), $\mathrm{T}_{1} / \mathrm{S}_{0}$ 交点构型在 $\mathrm{S}_{0}$ 态势能面 上最终落到 $\mathrm{S}_{0}$ 态平衡构型位置上(见图 6), $\mathrm{S}_{1}$ 与 $\mathrm{S}_{0}$ 的交点在 $\mathrm{S}_{0}$ 势能面上最终落到 $\mathrm{S}_{0}$ 态的平衡 构型位置上(见图 7). 另外, 垂直激发后, Franck-Condon 点在 $S_{1}$ 势能面上, 分子最终回到 $S_{1}$ 态

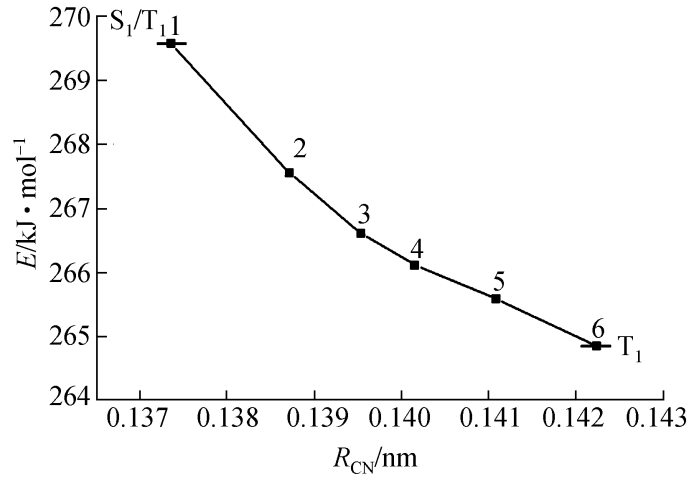

图 $5 \mathrm{~S}_{1} / \mathrm{T}_{1}$ 在 $\mathrm{T}_{1}$ 势能面上 经驰豫振动能量变化图

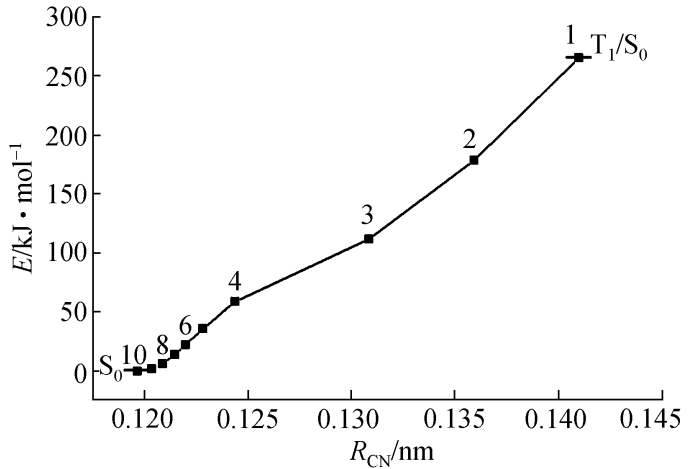

图 $6 \mathrm{~T}_{1} / \mathrm{S}_{0}$ 在 $\mathrm{S}_{0}$ 势能面上 经弛豫振动能量变化图 
的反式平衡构型(见图 8).

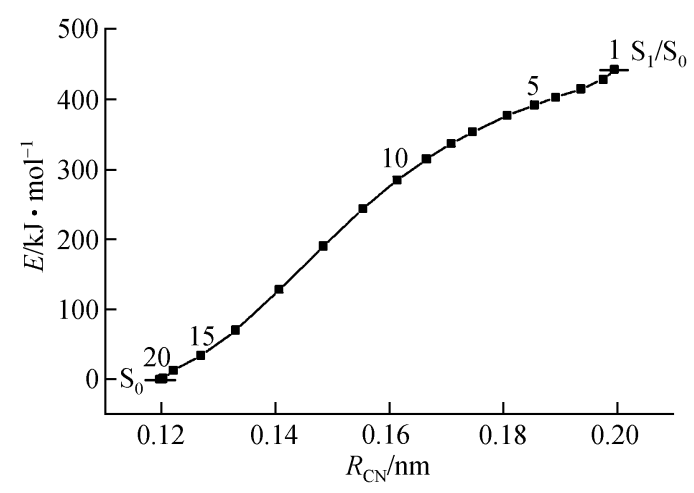

图 $7 \quad \mathrm{~S}_{1} / \mathrm{S}_{0}$ 在 $\mathrm{S}_{0}$ 势能面上 经驰豫振动能量变化图

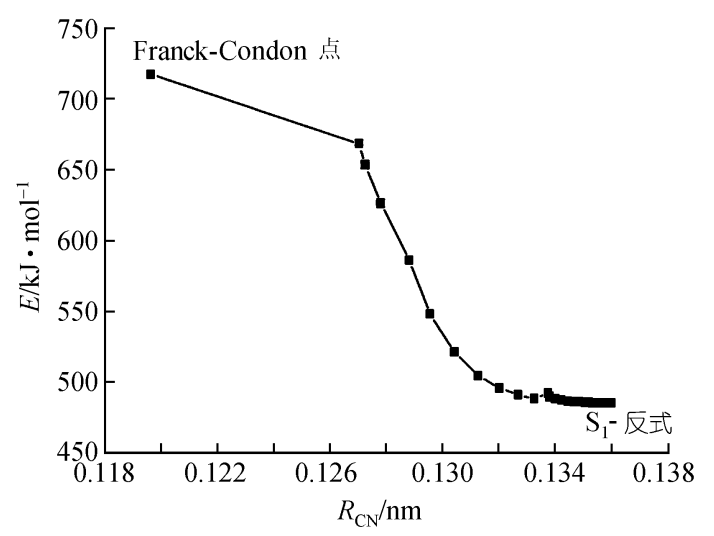

图 8 垂直激发后分子在 $S_{1}$ 势能面上 经驰豫振动能量变化图

这样我们就找到了不同电子态势能面的交叉点到低势能面平衡几何构型(稳定态)的演化 途径.

\section{4 小结}

对于 $\mathrm{HNCO} \stackrel{h v}{\longrightarrow} \mathrm{HN}+\mathrm{CO}$ 反应, 综合上述计算结果, 我们给出了反应进程的示意图(图 9).

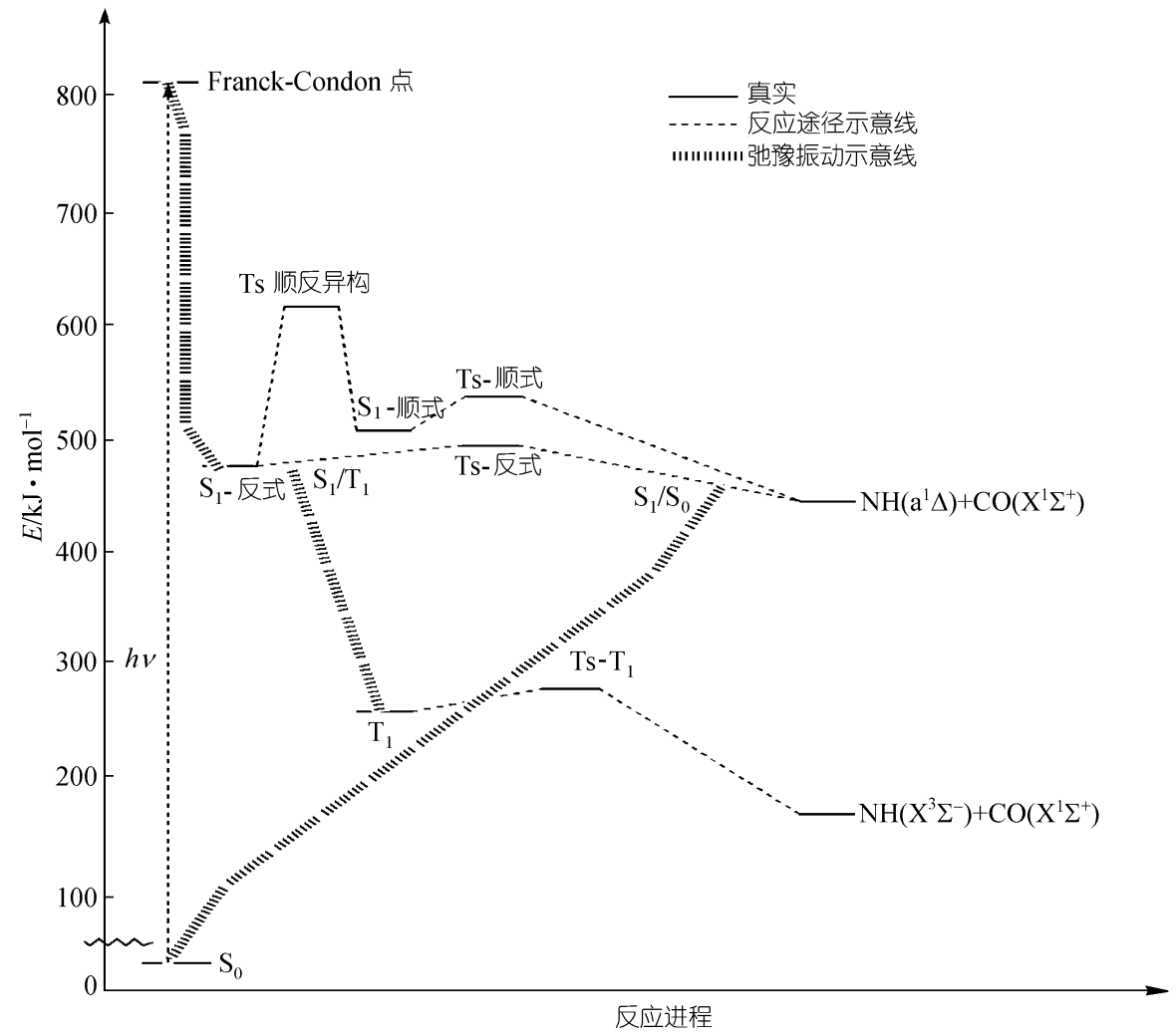

图 9 反应进程示意图 
从图 9 可知, 当异氧酸基态分子吸收一个光子, 电子被激发后, Franck-Condon 点经弛豫振 动最终落到 $\mathrm{S}_{1}$ 态反式平衡构型上, 此时异氰酸的光解反应 $\mathrm{HNCO} \stackrel{h v}{\longrightarrow} \mathrm{HN}+\mathrm{CO}$ 可能有 3 条解 离通道:

(A)

$$
\begin{aligned}
& \mathrm{S}_{1} \text { (反) } \rightarrow \mathrm{S}_{1} / \mathrm{T}_{1} \rightarrow \mathrm{T}_{1} \rightarrow \mathrm{Ts}_{1} \mathrm{~T}_{1} \rightarrow \mathrm{NH}\left(\mathrm{X}^{3} \Sigma^{-}\right)+\mathrm{CO}\left(\mathrm{X}^{1} \Sigma^{+}\right) \\
& \mathrm{NH}\left(\mathrm{a}^{1} \Delta\right)+\mathrm{CO}\left(\mathrm{X}^{1} \Sigma^{+}\right)
\end{aligned}
$$

显然从动力学的观点看, 通道 $(\mathrm{A})$ 对反应最为有利(即反应主要在 $\mathrm{T}_{1}$ 态上进行, 生成三态 产物), 通道(B)次之, $(\mathrm{C})$ 最弱. 这个结果和 Zyrianov 等推测这个反应为三态产物一致 ${ }^{[10]}$. 有关 这 3 个反应途径的竞争, 我们将在 $\mathrm{HNCO} \stackrel{h v}{\longrightarrow} \mathrm{HN}+\mathrm{CO}$ 动态学研究中讨论.

\section{参 考文献}

1 Brown S S, Berghout H L, Crim F F, Internal energy distribution of the NCO fragment from near-threshold photolysis of isocyanic acid HNCO. J Phys Chem, 1996, 100: 7948 7955

2 Zyrianov M, Droz-Georget Th, Reisler H. Competitive photodissociation channels in jet-cooled HNCO: Thermochemisty and near-threshold predissociation. J Chem Phys, 1996, 105(18): 8111 8116

3 Droz-Georget Th, Zyrianov M, Reisler H, et al. Correlated distributions in the photodissociation of $\mathrm{HNCO}$ to $\mathrm{NH}\left(\mathrm{X}^{3} \Sigma^{-}\right.$, $\left.\mathrm{a}^{1} \Delta\right)+\mathrm{CO}\left(\mathrm{X}^{1} \Sigma^{+}\right)$near the barrier on $\mathrm{S}_{1}$. Chem Phys Lett, 1997, 276: 316 324

4 Brown S S, Metz R B, Crim F F, et al. Vibrationally mediated photodissociation of isocyanic acid (HNCO): Preferential N-H bond fission by excitation of the reaction coordinate. J Chem Phys, 1995, 105(15): 6293 6303

5 Brown S S, Berghout H L, Crim F F. Vibrational state controlled bond cleavage in the photodissociation of isocyanic acid (HNCO). J Chem Phys, 1995, 102(21): 8440 8447

6 Steven J E, Qiang Cui, Morokuma K. An ab initio study of the dissociation of HNCO in the $\mathrm{S}_{1}$ electronic state. J Chem Phys, 1998, 108(4): 1452 1458

7 Fang Weihai, You Xiaozeng, Zhen Yin. Theoretical studies on photolysis and pyrolysis of isocyanic acid. Chem Phys Lett, 1995, 238: 236 242

Brown S S, Cheatum C M , Crim F F, et al. A simple model of the $\operatorname{HNCO}\left({ }^{1} \mathrm{~A}^{\prime}\right)$ excited state potential energy surface and a classical trajectory analysis of the vibrationally directed bond-selected photodissociation. J Chem Phys, 1996, 105(24): 10911 10918

9 Klossica J J, Lothmann H F, Yamashita K. The topography of the $\operatorname{HNCO}\left(\mathrm{S}_{1}\right)$ potential energy surface and its implication for photodissociation dynamics. Chem Phys Lett, 1997, 276: 325 333

10 Zyrianov M, Droz-Georget Th, Reisler H. Fragment recoil anisotropies in the photoinitiated decomposition of HNCO. J Chem Phys, 1999, 110(4): 2059 2068

11 Koga N, Morokuma K. Determination of the lowest energy point on the crossing seam between two potential surfaces using the energy gradient. Chem Phys Lett, 1985, 119: 371 374 